\title{
Therapeutic potential of antisense oligonucleotides as modulators of alternative splicing
}

\author{
Peter Sazani and Ryszard Kole \\ Lineberger Comprehensive Cancer Center and Department of Pharmacology, University of North Carolina, \\ Chapel Hill, North Carolina, USA
}

\begin{abstract}
An estimated $60 \%$ of all human genes undergo alternative splicing, a highly regulated process that produces splice variants with different functions. Such variants have been linked to a variety of cancers, and genetic diseases such as thalassemia and cystic fibrosis. This Perspective describes a promising approach to RNA repair based on the use of antisense oligonucleotides to modulate alternative splicing and engender the production of therapeutic gene products.
\end{abstract}

J. Clin. Invest. 112:481-486 (2003). doi:10.1172/JCI200319547.

The use of antisense 2 -deoxyoligonucleotides as agents for specific downregulation of gene expression is now well established (1). Most commonly, the oligonucleotides base-pair with an mRNA target and mediate its destruction by RNase $\mathrm{H}$, an enzyme that destroys the RNA in a DNA/RNA duplex. Numerous clinical trials evaluating the use of downregulating antisense oligonucleotides for the treatment of cancer and other diseases are now underway (2). The oligonucleotide drug Vitravene, the first of such drugs to be approved, is used to treat cytomegalovirus retinitis. More recently, antisense compounds are being developed for manipulation of alternative splicing patterns. In this way, the ratio of different splice variants can be altered, and the function of a gene changed. Considering that there are approximately 30,000 human genes and that up to $60 \%$ are alternatively spliced, this new antisense approach may have far-reaching implications in the treatment of a variety of diseases. Oligonucleotides can be used to silence mutations that cause aberrant splicing, thus restoring correct splicing and function of the defective gene.

\footnotetext{
Address correspondence to: Ryszard Kole, University of North Carolina, Lineberger Comprehensive Cancer Center, CB \#7295, Chapel Hill, North Carolina 27599-7295, USA.

Phone: (919) 966-1143; Fax: (919) 966-3015;

E-mail:kole@med.unc.edu.

Conflict of interest: Peter Sazani, who left the laboratory in December 2002, has no conflicts of interest. Ryszard Kole is a founder of Ercole Biotech Inc., a company that exploits Ryszard Kole's intellectual property on modification of alternative splicing by antisense oligonucleotides. Research in Ryszard Kole's laboratory is supported in part via a sponsored research agreement between the University of North Carolina and Ercole Biotech Inc.

Nonstandard abbreviations used: 2'-O-methoxyethyl (2'-OMOE); peptide nucleic acid (PNA); intron 2 (IVS2); cystic fibrosis transmembrane conductance regulator (CFTR); Duchenne muscular dystrophy (DMD); IL-5 receptor (IL-5R); human insulin receptor (HIR); polycystic kidney disease (PKD); hepatic lipase (HL); prostate-specific membrane antigen (PSM); enhanced GFP (EGFP).
}

This is important since close to $50 \%$ of genetic disorders are caused by mutations that cause defects in premRNA splicing. Thus, targeting splicing with antisense oligonucleotides significantly extends the clinical potential of these compounds.

The requirements for oligonucleotides that shift splicing are different from those for oligonucleotides used to achieve downregulation. Specifically, they must not activate RNase $\mathrm{H}$, which would destroy the pre-mRNA target before it could be spliced. They must also be able to effectively compete with splicing factors for access to target pre-mRNAs that reside within the nuclei of cells. Several types of modified synthetic oligonucleotides fit these criteria. For example, oligonucleotides with modifications to the $2^{\prime}$ position, such as 2'-O-methyl, 2'-O-methoxyethyl (2'-O-MOE) and 2'$\mathrm{O}$-aminopropyl, are $\mathrm{RNase} \mathrm{H}$ inactive and generally have higher nuclease resistance and affinity for target sequences than their 2'-deoxy counterparts. Oligonucleotides with backbones based on morpholino, peptide nucleic acid (PNA), phosphoramidate and methylphosphonate derivatives of nucleotides have similar characteristics (2). Modifications to the bases, which increase the affinity of oligonucleotides for their target, may facilitate favorable antisense activity in the context of splicing (2). The above modifications represent the second or third generation of compounds superior to the initially extensively studied phosphorothioate oligodeoxynucleotides, which frequently exhibited nonspecific, non-antisense effects (1).

\section{Correction of splicing by antisense oligonucleotides}

$\beta$-Globin. $\beta$-Thalassemia is a serious genetic blood disorder (3) in which production of $\beta$-globin, a subunit of hemoglobin, is partially or entirely ablated by mutations in the $\beta$-globin gene. The defect decreases the oxygen-carrying capacity of red blood cells and causes compensatory expansion of the bone marrow 
a

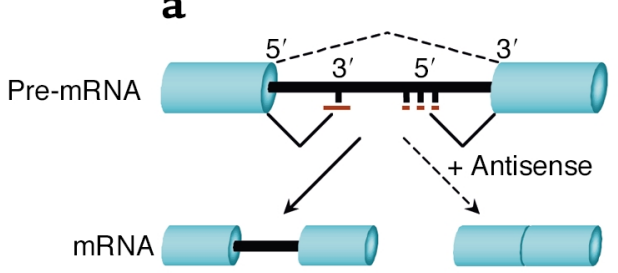

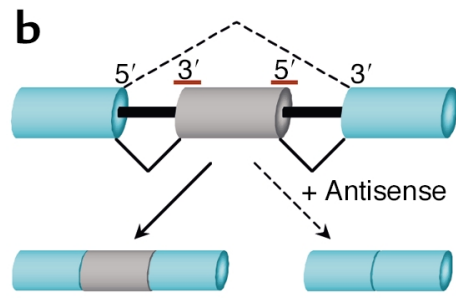

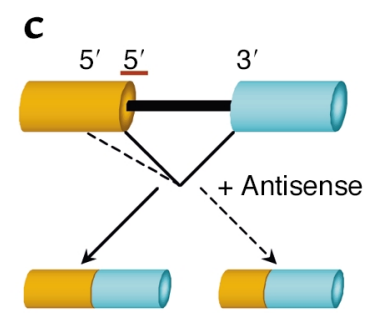

\section{Figure 1}

Modification of splicing by antisense oligonucleotides. Aberrant splicing in thalassemic $\beta$-globin pre-mRNA or in certain splice mutants in CFTR is prevented, and correct splicing is restored, by oligonucleotides (dark red bars) that block aberrant $5^{\prime}$ or $3^{\prime}$ cryptic splice sites (a). Similarly, oligonucleotides induce skipping of a normal exon (gray) (b) or force selection of an alternative $5^{\prime}$ splice site (c) by antisense oligonucleotides targeted to appropriate splice sites.

and, if untreated, fatal iron overload. Mutations that cause splicing defects are common among thalassemic patients. For example, mutations at position 654,705 , or 745 of intron 2 (IVS2) of human $\beta$-globin pre-mRNA lead to creation of an aberrant $5^{\prime}$ splice site and activation of a cryptic $3^{\prime}$ splice site within the intron (Figure 1a). The result is the inclusion of part of IVS2 (creating an in-frame stop codon) into the spliced mRNA. As a potential therapeutic, RNase $\mathrm{H}$-inactive antisense oligonucleotides targeted to the aberrant splice site block its use by the spliceosome, restoring the use of the proper splice sites and thus $\beta$-globin expression (Figure 1a).

Ex vivo correction of $\beta$-globin pre-mRNA was accomplished in human erythroid progenitor cells (4). Correction of splicing and upregulation of the $\beta$-globin protein were seen at concentrations of up to 45 $\mu \mathrm{M}$ morpholino oligomer delivered by temporary mechanical disruption of the cells. Despite high doses, sequence specificity was maintained and treatment appeared generally nontoxic. Recent results show that prolonged free uptake of morpholino oligonucleotides also effectively corrects splicing in these cells (5). Intracellular localization studies confirmed that the antisense oligonucleotides must have entered the nucleus in order to effectively modulate splicing. Another $\beta$-globin mutation, $\beta^{\mathrm{E}}$, was also successfully targeted using this approach (6). In an alternative and potentially longer-term strategy, the antisense sequences were incorporated into lentiviral expression vectors and used to successfully treat human erythropoietic progenitor cells ex vivo (7).

Cystic fibrosis transmembrane conductance regulator. A mutation $(3849+10 \mathrm{~kb}$ C to T) in intron 19 of the cystic fibrosis transmembrane conductance regulator (CFTR) gene also creates an aberrant $5^{\prime}$ splice site and activates a cryptic $3^{\prime}$ splice site (see Figure 1a). This splicing defect accounts for roughly $2 \%$ of all cystic fibrosis cases but is more frequent in the Ashkenazi Jewish population. Treatment of cell lines expressing this form of CFTR with antisense 2'-O-methyl oligonucleotides targeted to either the $5^{\prime}$ or $3^{\prime}$ aberrant splice sites leads to dose-dependent increases in CFTR protein expression (8). Interestingly, delivery of both oligonucleotides simultaneously had an additive effect, a result not seen with $\beta$-globin (R. Kole, unpublished observations). Other splicing defect mutations also occur in CFTR (9), representing additional potential targets for the antisense approach.

Dystrophin. Mutations that create stop codons in the dystrophin gene are the main cause of Duchenne muscular dystrophy (DMD). In contrast, a milder form of the disease, Becker muscular dystrophy, results from in-frame deletions. It was hypothesized that oligonucleotides could be used to cause skipping of the exon containing the stop codon, restoring the reading frame and thus partially ameliorating the DMD disease state (Figure 1b). Cells derived from the $m d x$ mouse model of DMD (containing the stop codon in dystrophin exon 23) were treated with $2^{\prime}$-Omethyl oligonucleotides targeted to the $5^{\prime}$ splice site of the exon. This treatment caused exclusion of exon 23 from the spliced $m d x$ message, thus reducing its length by 213 nucleotides and eliminating the premature stop codon (10).

More recent work showed that formulations of the same oligonucleotides injected directly into the $m d x$ mouse muscle modified splicing, restored the dystrophin reading frame (11), and increased the strength of the injected muscle (12). Other studies have found that targeting a splicing enhancer in another region of the gene can also produce an exon-skipping effect in cultured myotubes from DMD patients, and that a skipping rate of only $15 \%$ was sufficient to restore properly localized dystrophin to $75 \%$ of normal levels in most cells $(12,13)$. The antisense oligonucleotidedriven shift in splicing may be particularly attractive as a treatment for DMD, since the dystrophin gene is very large $(2,400 \mathrm{~kb})$ and therefore difficult to deliver by current gene therapy protocols.

Tau. The tau gene codes for six splice variants of a neuron-specific microtubule-associated protein. In human neurons, exon 10 is normally skipped, because a hairpin structure in the pre-mRNA sequesters its $5^{\prime}$ splice site. Point mutations that disrupt the hairpin lead to inclusion of exon 10 into the mRNA, causing a disorder manifested by frontotemporal dementia and parkinsonism linked to chromosome 17 (known as FTDP-17), with 
symptoms similar to those of Alzheimer disease. Two rat cell lines, which express tan with exon 10, were used as models for this splicing defect. Kalbfuss et al. delivered 2'-O-methyl oligonucleotide antisense to the damaged hairpin structure to these cells in the hopes of restoring the skipping of exon 10 (14). This treatment resulted in a dose-dependent increase in the proportion of tan that was devoid of exon 10 (Figure 1b). Correction of splicing was very sensitive, with an $\mathrm{EC}_{50}$ of approximately $1.5 \mathrm{nM}$ (effective concentration of the oligonucleotide shifting splicing by $50 \%$ ) when oligonucleotides were delivered by electroporation. The treatment also caused alterations in cell morphology as a result of cytoskeletal-structure rearrangement, a therapeutically favorable outcome.

\section{Modification of alternative splicing by antisense oligonucleotides}

In the above examples, gene function rendered defective by the splicing mutations was restored by antisense oligonucleotides. Examples below demonstrate modification of natural alternative splicing or induction of aberrant splicing that promises clinically relevant outcomes.

$B c l-x$. Bcl-x codes for two splice variants with opposing functions that are derived by the use of two alternative $5^{\prime}$ splice sites. The long form, bcl-xL, has antiapoptotic properties, while the short form, bcl-xS, has proapoptotic properties. Both forms are required for the normal cellular function; however, bcl-xL is over-represented in a number of cancers, including prostate cancer (15). Therefore, blocking the $\mathrm{xL}$ (proximal) $5^{\prime}$ splice site should increase the ratio of bcl-xS to bcl-xL and thereby simultaneously increase proapoptotic signals, decrease antiapoptotic signals, and inhibit the growth or cause the death of the cancer cells (Figure 1c).

Taylor et al. targeted the sequence adjacent to the $\mathrm{xL}$ splice site with 2 '-O-MOE phosphorothioate oligonucleotide delivered by cationic lipids to A549 lung carcinoma cells (16). A dose-dependent and sequence-specific increase in bcl-xS expression was seen, with optimal shifting occurring at 24 hours and 100-200 nM oligonucleotide. The newly synthesized bcl-xS transcripts were successfully translated into protein. The resulting proapoptotic signal did not cause cell death but sensitized the A549 cells to apoptosis by cis-platinum. Interestingly, Mercatante et al. demonstrated that an antisense 2'$\mathrm{O}$-methyl oligonucleotide targeted to the $\mathrm{xL}$ splice site alone induced apoptosis in PC3 prostate cancer cells $(17,18)$. Here, maximal shifting of splicing occurred with $80 \mathrm{nM}$ oligonucleotide approximately 16-24 hours after delivery by cationic lipid transfection. Increases in bcl-xS protein translation peaked at 24-36 hours after treatment, suggesting that the antisense effects are relatively long lasting. Additional experiments showed that shifting of the balance between bcl-xS and bcl-xL induces apoptosis in PC3 cells more effectively than does downregu- lation of bcl-xL. Recent work indicates that the level of expression of bcl-x gene is an important determinant of cell sensitivity to bcl-x oligonucleotide treatment (18).

$I L-5$ receptor. A membrane-bound or a soluble form of the IL-5 receptor (IL-5R) is generated by alternative splicing and respective inclusion or exclusion of exon 9 , which codes for the transmembrane domain of the protein (19). The latter form may represent a decoy receptor with no physiological function other than to prevent signaling by IL-5. Therefore, preventing the inclusion of exon 9 should not only decrease the number of functional IL-5Rs but also lead to a further decrease in the available concentration of the agonist, IL-5. This approach may have therapeutic effects on diseases of inflammation, such as asthma. Antisense 2'-O-MOE oligonucleotides targeted to regions spanning exon 9 were delivered to $B$ cell lymphoma cells by electroporation (20). As expected, an increase in the soluble IL-5R with a concomitant decrease in the insoluble form was observed (see Figure 1b), suggesting that the alternative splicing pathway of IL-5R was manipulated. This notion was confirmed by treatment of cells with an RNase $\mathrm{H}$-competent oligonucleotide targeted to the same region. This time, both forms of the receptor were downregulated. Considering that the mRNA coding for the soluble form does not contain the target sequence, the result strongly suggests that the nuclear pre-mRNA was the primary target of RNase H-mediated destruction (see "Evaluation of next-generation antisense oligonucleotides").

$\mathrm{C}-m y c$. C-myc is a powerful oncogene that is overexpressed or mutated in several types of cancer. Application of antisense oligonucleotides for downregulation of c-myc by a 28 -mer morpholino oligomer directed toward the AUG codon was unexpectedly shown to cause aberrant splicing (21). It was found that, in addition to AUG, the oligonucleotide blocked the intron/ exon junction of exon 2, activating a cryptic 3 ' splice site further upstream within the exon. Thus, the region of exon 2 containing the start codon was omitted from the spliced message, leading to translation initiation from a downstream AUG and generating a truncated c-myc protein. This protein was detectable with a c-myc antibody and did not appear when cells were treated by random or sense control oligomers.

\section{Clinically relevant potential targets for antisense oligonucleotides that shift splicing}

Following the sequencing of the human genome, the discrepancy between the number of genes and the number of gene products suggests that a large number of the latter are generated by alternative splicing. Thus, manipulation of alternative splicing may have widespread applications. Discussed below are several examples of potential targets for this approach.

Human insulin receptor. The gene encoding the human insulin receptor (HIR) is expressed as two different tissue-specific splice variants, HIR-A and HIR-B, that exclude or include exon 11, respectively. 


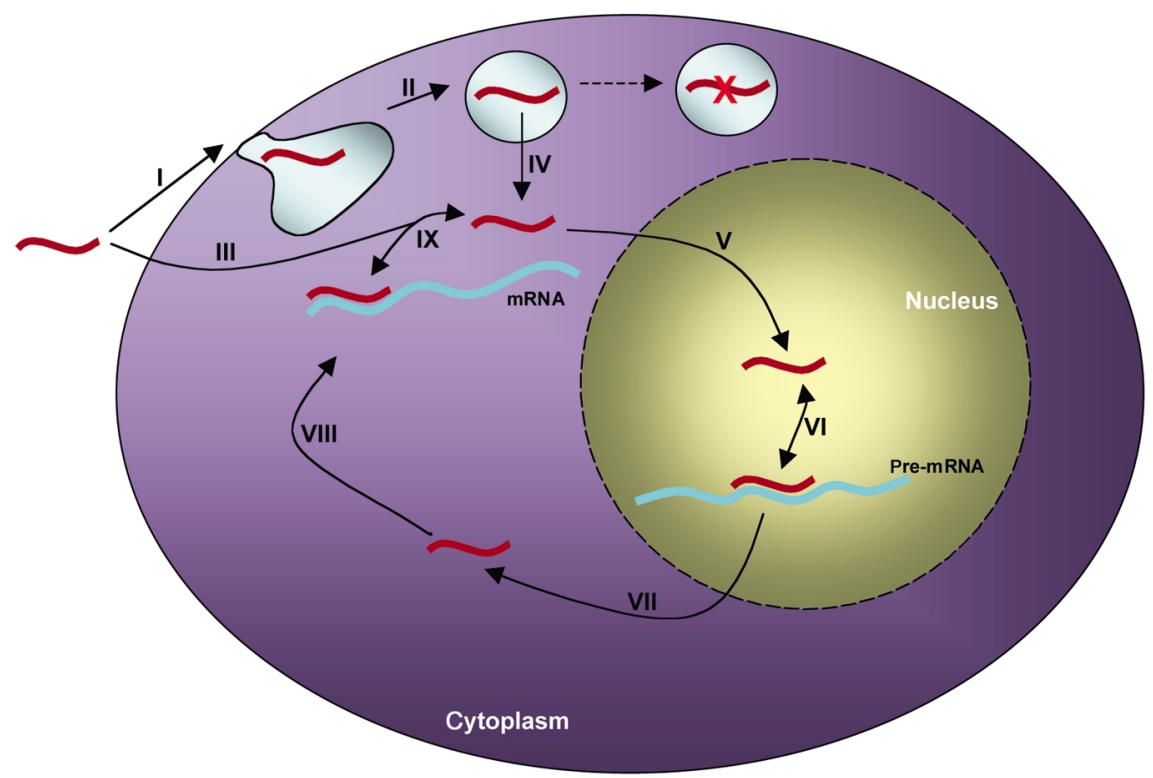

\section{Figure 2}

Site of action of antisense oligonucleotide. (I) From the extracellular space, an oligonucleotide or oligonucleotide/cationic lipid complex interacts with the cell surface, presumably through receptor-ligand binding. (II) The oligonucleotide then undergoes endocytosis and is thus sequestered from the rest of the cytoplasm. Alternatively (III) an oligonucleotide conjugated to cell-penetrating peptide fragments may enter the cell via a nonendocytotic mechanism. (IV) Escape from the endosome is facilitated by the cationic lipid. Oligonucleotides that do not escape are degraded in the lysosome (dashed arrow). (V) Once free in the cytoplasm, the oligonucleotide can passively diffuse into the nucleus, where (VI) hybridization to the pre-mRNA target can take place. (VII) Export from the nucleus is energy dependent and involves interactions of the oligonucleotide with nuclear export factors, such as the nuclear pore complex. (VIII) The biologically active oligonucleotide can then bind cytoplasmic mRNA. (IX) The oligonucleotide can also dissociate from the mRNA target and recycle.

It has been reported that HIR-A binds insulin with higher affinity than does HIR-B but has lower signaling capacity than the latter. Although the conclusions are somewhat conflicting (22), it appears that the levels of inclusion of exon 11 in tissues may contribute to type 2 diabetes. Manipulation of the ratio between the splice variants may better elucidate their functions and possibly result in therapeutic applications of oligonucleotides. It appears that there are exonic and intronic regions in the HIR premRNA that either negatively or positively influence exon 11 splicing (23). Blocking of these regions as well as the splice sites flanking the exon by antisense oligonucleotides should allow for control over which variant is expressed.

$G A B A_{A} \gamma 2$. The $G A B A_{A} \gamma 2$ gene is alternatively spliced to generate a short $(\gamma 2 S)$ and a long $(\gamma 2 L)$ form. It was recently shown that the ratio of $\gamma 2 \mathrm{~S}$ to $\gamma 2 \mathrm{~L}$ is severely reduced in the prefrontal cortex of schizophrenia patients (24) and in animal models of alcoholism (25). This suggests that reduction of the levels of the long form, in addition to increases in the levels of the short form, could be of clinical benefit (Figure 1b). The alternatively spliced exon is only $24 \mathrm{bp}$, allowing for complete targeting by an antisense oligonucleotide that, theoretically, would promote skipping and improve the ratio of $\gamma 2 \mathrm{~S}$ to $\gamma 2 \mathrm{~L}$.

Fibronectin. Fibronectin is an ECM protein critical for the maintenance of normal skin. Different forms of fibronectin can arise by alternative splicing of three domains, known as EDA, EDB, and IIICS. One form, called $\mathrm{EDA}^{+} \mathrm{Fn}$, includes the EDA domain and is thought to be involved in the development of psoriasis (26), an immune and hyperproliferation disorder of the skin. Specifically, an increased ratio of $\mathrm{EDA}^{+} \mathrm{Fn}$ to $\mathrm{EDA}^{-}$Fn was found to be expressed in the epidermis of psoriasis patients but not in controls, suggesting the occurrence of a clinically relevant alternative splicing event. Thus, oligonucleotide-induced skipping of the EDA exon may ameliorate the disease (Figure $1 \mathrm{~b}$ ).

Polycystic kidney disease. Polycystic kidney disease (PKD) is among the most common inherited disorders, with three genes (PKD1, PKD2, and PKD3) known to be involved in its pathogenesis (27). One known point mutation in $P K D 2$ activates a cryptic $5^{\prime}$ splice site within exon 14 that is exclusively utilized in preference to the still potentially functional, authentic site (28). Interestingly, the activated splice site is 24 bp upstream of the mutation. Antisense oligonucleotides targeted to either the point mutation or the aberrant $5^{\prime}$ splice site may be sufficient to restore correct splicing, thus restoring $P K D 2$ gene function.

Hepatic lipase. Deficiency in hepatic lipase (HL) leads to hypertriglyceridemia and premature cardiovascular disease. A mutation in intron 1 of HL causes generation of a new $3^{\prime}$ splice site, which leads to a prematurely truncated aberrant transcript and loss of HL function (29). 
The new splice site is completely dominant, with no correctly spliced message observed in the patient. Antisense oligonucleotides targeted to this defect should restore proper splicing. Though this mutation is currently thought to be uncommon, targeting of this and other defects in HL with antisense compounds could have great clinical benefit, considering that pharmacokinetic data show the liver to be a favorable target for oligonucleotide therapy $(30,31)$.

Prostate-specific membrane antigen. Prostate-specific membrane antigen (PSM) has a long and a short splicing isoform (PSM and $\mathrm{PSM}^{\prime}$ ) generated by the use of alternative $3^{\prime}$ splice sites in exon 3. The PSM/PSM' ratio is about 150 times higher in prostate cancer relative to normal tissue and may constitute a marker for the emergence and progression of the disease (32). Although there is no direct evidence that the long, PSM form causes cancer, recent reports suggest that PSM may be a valuable therapeutic target $(33,34)$. Thus it would be of interest to determine the biological effects of oligonucleotide-induced exon skipping and restoration of the correct $\mathrm{PSM} / \mathrm{PSM}^{\prime}$ ratio in prostate cancer tissue.

\section{Evaluation of next-generation antisense oligonucleotides}

Downregulation of gene expression by $\mathrm{RNase} \mathrm{H}$-competent oligonucleotides targeted to the AUG codon or other sequences in mRNA is a predominant method of utilizing these compounds (1). Reports also indicate that targeting the AUG start codon with RNase $\mathrm{H}$-inactive oligonucleotides could successfully inhibit gene expression (35). Thus it seemed evident that the mRNA located in the cytoplasm had to be the intracellular site of action of these compounds. However, closer evaluation of the data strongly suggests that the nucleus is actually the predominant site of action of antisense molecules (Figure 2). Early reports on antisense delivery agents showed that the marked increase in efficacy of antisense molecules correlated with their increased nuclear accumulation (36-38). Furthermore, in the successfully targeted genes listed above, the alteration of splicing patterns had to have resulted from the binding of the oligonucleotide to the nuclear pre-mRNA. Thus, it is clear that a mechanism for evaluating the sequence-specific efficacy of novel antisense compounds must reflect the nuclear antisense activity of the compound.

An assay for the nuclear activity of antisense oligonucleotides was developed, based on the correctable aberrant splicing of IVS2-654 human $\beta$-globin intron 2 . This intron was inserted into the coding sequence for enhanced GFP (EGFP) and thus prevented expression of EGFP both in stable HeLa cell lines (39) and in a transgenic mouse that ubiquitously expressed the EGFP-654 construct (31). Upregulation of EGFP was accomplished by antisense oligonucleotides directed at the aberrant $5^{\prime}$ splice site at position 654 in the intron. Increases in EGFP pro- duction were proportional to antisense activity of a given oligonucleotide. Using these systems, RNase $\mathrm{H}$-inactive antisense compounds with novel or established chemistry can be compared in both the cellular and the animal model, on a standard platform with a standardized sequence. Therefore, the variability in sequence and output associated with different assays is eliminated. Initial studies using the assay found that negatively charged 2 '-O-MOE phosphorothioate and positively charged PNA-4K oligomers (PNA with four positively charged lysine residues at the carboxy $\left[3^{\prime}\right]$ end) had superior activity in the transgenic mouse compared with uncharged oligomers such as morpholino and PNA-1K (with one lysine). In cell culture, free uptake of these oligomers was dependent on a lack of negative charge or the presence of a positive charge. These results demonstrate an apparent inability of cell culture to predict in vivo efficacy; however, in both instances, the positively charged oligomers (e.g., PNA-4K) were most active. More work is needed to optimize antisense oligonucleotides for in vivo and clinical application, and the transgenic EGFP-654 mouse provides a useful tool for these experiments.

\section{Acknowledgments}

The work in this laboratory was supported in part by grants HL-51940 and PO1-GM59299 from the NIH and through a research agreement with Ercole Biotech Inc.

1. Crooke, S.T. 2001. Antisense drug technology: principles, strategies and applications. Marcel Dekker Inc. New York, New York, USA. 916 pp.

2. Mercatante, D., Sazani, P., and Kole, R. 2001. Modification of alternative splicing pathways as a potential chemotherapy for cancer and other diseases. Curr. Cancer Drug Targets. 1:211-230.

3. Schwartz, E., Benz, Jr., E.J., and Forget, B.G. 2000. Thalassemia syndromes. In Hemalotogy: basic principles and practice. 3 rd edition. R. Hoffman, et al., editors. Churchill Livingstone. New York, New York, USA. 485-510.

4. Lacerra, G., et al. 2000. Restoration of hemoglobin A synthesis in erythroid cells from peripheral blood of thalassemic patients. Proc. Natl. Acad. Sci. U. S. A. 97:9591-9596.

5. Suwanmanee, T., et al. 2002. Restoration of human beta-globin gene expression in murine and human IVS2-654 thalassemic erythroid cells by free uptake of antisense oligonucleotides. Mol. Pharmacol. 62:545-553.

6. Suwanmanee, T., Sierakowska, H., Fucharoen, S., and Kole, R. 2002. Repair of a splicing defect in erythroid cells from patients with $\beta$-thalassemia/HbE disorder. Mol. Ther. 6:718-726.

7. Vacek, M.M., et al. 2003. High-level expression of hemoglobin A in human thalassemic erythroid progenitor cells following lentiviral vector delivery of an antisense snRNA. Blood. 101:104-111.

8. Friedman, K.J., et al. 1999. Correction of aberrant splicing of the cystic fibrosis transmembrane conductance regulator (CFTR) gene by antisense oligonucleotides. J. Biol. Chem. 274:36193-36199.

9. Tsui, L.C. 1992. The spectrum of cystic fibrosis mutations. Trends Genet. 8:392-398.

10. Wilton, S.D., et al. 1999. Specific removal of the nonsense mutation from the mdx dystrophin mRNA using antisense oligonucleotides. Neuromuscul. Disord. 9:330-338.

11. Mann, C.J., et al. 2001. Antisense-induced exon skipping and synthesis of dystrophin in the $\mathrm{mdx}$ mouse. Proc. Natl. Acad. Sci. U. S. A. 98:42-47.

12. Lu, Q.L., et al. 2003. Functional amounts of dystrophin produced by skipping the mutated exon in the mdx dystrophic mouse. Nat. Med. doi: $10.1038 / \mathrm{nm} 897$.

13. van Deutekom, et al. 2001. Antisense-induced exon skipping restores dystrophin expression in DMD patient derived muscle cells. Hum. Mol. Genet. 10:1547-1554.

14. Kalbfuss, B., Mabon, S.A., and Misteli, T. 2001. Correction of alterna- 
tive splicing of tau in frontotemporal dementia and parkinsonism linked to chromosome 17. J. Biol. Chem. 276:42986-42993.

15. Krajewska, M., et al. 1996. Immunohistochemical analysis of bcl-2, bax bcl-X, and mcl-1 expression in prostate cancers. Am. J. Pathol. 148:1567-1576.

16. Taylor, J.K., Zhang, Q.Q., Wyatt, J.R., and Dean, N.M. 1999. Induction of endogenous Bcl-xS through the control of Bcl-x pre-mRNA splicing by antisense oligonucleotides. Nat. Biotechnol. 17:1097-1100.

17. Mercatante, D.R., Bortner, C.D., Cidlowski, J.A., and Kole, R. 2001 Modification of alternative splicing of $\mathrm{Bcl}-\mathrm{x}$ pre-mRNA in prostate and breast cancer cells. Analysis of apoptosis and cell death. J. Biol. Chem. 276:16411-16417.

18. Mercatante, D.R., Mohler, J.L., and Kole, R. 2002. Cellular response to an antisense-mediated shift of Bcl-x pre-mRNA splicing and antineoplastic agents. J. Biol. Chem. 277:49374-49382.

19. Kotsimbos, A.T., and Hamid, Q. 1997. IL-5 and IL-5 receptor in asthma. Mem. Inst. Oswaldo Cruz. 92:75-91.

20. Karras, J.G., McKay, R.A., Dean, N.M., and Monia, B.P. 2000. Deletion of individual exons and induction of soluble murine interleukin-5 receptor-alpha chain expression through antisense oligonucleotidemediated redirection of pre-mRNA splicing. Mol. Pharmacol. 58:380-387.

21. Giles, R.V., Spiller, D.G., Clark, R.E., and Tidd., D.M. 1999. Antisense morpholino oligonucleotide analog induces missplicing of C-myc mRNA. Antisense Nucleic Acid Drug Dev. 9:213-220.

22. Sesti, G. 2000. Insulin receptor variant forms and type 2 diabetes mellitus. Pharmacogenomics. 1:49-61.

23. Kosaki, A., Nelson, J., and Webster, N.J. 1998. Identification of intron and exon sequences involved in alternative splicing of insulin receptor pre-mRNA. J. Biol. Chem. 273:10331-10337.

24. Huntsman, M.M., Tran, B.V., Potkin, S.G., Bunney, Jr., W.E., and Jones, E.G. 1998. Altered ratios of alternatively spliced long and short gamma2 subunit mRNAs of the gamma-amino butyrate type A receptor in prefrontal cortex of schizophrenics. Proc. Natl. Acad. Sci. U. S. A. 95:15066-15071.

25. Petrie, J., et al. 2001. Altered gabaa receptor subunit and splice variant expression in rats treated with chronic intermittent ethanol. Alcohol. Clin. Exp. Res. 25:819-828.

26. Ting, K.M., et al. 2000. Overexpression of the oncofetal Fn variant con- taining the EDA splice-in segment in the dermal-epidermal junction of psoriatic uninvolved skin. J. Invest. Dermatol. 114:706-711.

27. Grantham, J.J., and Calvet, J.P. 2001. Polycystic kidney disease: in danger of being X-rated? Proc. Natl. Acad. Sci. U. S. A. 98:790-792.

28. Reynolds, D.M., et al. 1999. Aberrant splicing in the PKD2 gene as a cause of polycystic kidney disease. J. Am. Soc. Nephrol. 10:2342-2351.

29. Brand, K., Dugi, K.A., Brunzell, J.D., Nevin, D.N., and SantamarinaFojo, S. 1996. A novel $A \rightarrow G$ mutation in intron $I$ of the hepatic lipase gene leads to alternative splicing resulting in enzyme deficiency. J. Lipid Res. 37:1213-1223.

30. Geary, R.S., et al. 2001. Pharmacokinetic properties of 2'-O-(2methoxyethyl)-modified oligonucleotide analogs in rats. J. Pharmacol. Exp. Ther. 296:890-897.

31. Sazani, P., et al. 2002. Systemically delivered antisense oligomers upregulate gene expression in murine tissues. Nat. Biotechnol. 20:1228-1233.

32. Kawakami, M., and Nakayama, J. 1997. Enhanced expression of prostate-specific membrane antigen gene in prostate cancer as revealed by in situ hybridization. Cancer Res. 57:2321-2324.

33. Tasch, J., Gong, K., Sadelain, M., and Heston, W.D. 2001. A unique folate hydrolase, prostate-specific membrane antigen (PSMA): a target for immunotherapy? Crit. Rev. Immunol. 21:249-261.

34. Stamey, T.A., et al. 2001. Molecular genetic profiling of Gleason grade $4 / 5$ prostate cancers compared to benign prostatic hyperplasia. J. Urol. 166:2171-2177.

35. Summerton, J. 1999. Morpholino antisense oligomers: the case for an RNase H-independent structural type. Biochim. Biophys. Acta. 1489:141-158.

36. Bennett, C.F., Chiang, M.Y., Chan, H., Shoemaker, J.E., and Mirabelli, C.K. 1992. Cationic lipids enhance cellular uptake and activity of phosphorothioate antisense oligonucleotides. Mol. Pharmacol. 41:1023-1033.

37. Zelphati, O., and Szoka, Jr., F.C. 1996. Intracellular distribution and mechanism of delivery of oligonucleotides mediated by cationic lipids. Pharm. Res. 13:1367-1372.

38. Marcusson, E.G., Bhat, B., Manoharan, M., Bennett, C.F., and Dean, N.M. 1998. Phosphorothioate oligodeoxyribonucleotides dissociate from cationic lipids before entering the nucleus. Nucleic Acids Res. 26:2016-2023

39. Sazani, P., et al. 2001. Nuclear antisense effects of neutral, anionic and cationic oligonucleotide analogs. Nucleic Acids Res. 29:3965-3974. 